\title{
Suppression of Ischemia-Induced Hippocampal Pyramidal Neuron Death by Hyaluronan Tetrasaccharide through Inhibition of Toll-Like Receptor 2 Signaling Pathway
}

\author{
Takehiko Sunabori, ${ }^{* \dagger}$ Masato Koike, ${ }^{*}$ Akira Asari, ${ }^{\ddagger}$ Yoji Oonuki, ${ }^{\S}$ and Yasuo Uchiyama
}

\begin{abstract}
From the Departments of Cell Biology and Neuroscience* and Cellular and Molecular Neuropathology, ${ }^{\dagger}$ Juntendo University Graduate School of Medicine, Bunkyo-Ku, Tokyo, Japan; the Department of Biomedical Engineering (ND20), ${ }^{\ddagger}$ Lerner Research Institute, Cleveland Clinic Foundation, Cleveland, Ohio; and Glycoscience Laboratories Inc.,,$^{\S}$ Tokyo, Japan
\end{abstract}

\author{
Accepted for publication \\ March 28, 2016. \\ Address correspondence to \\ Yasuo Uchiyama, M.D., Ph.D., \\ Department of Cellular and \\ Molecular Neuropathology, \\ Juntendo University Graduate \\ School of Medicine, 2-1-1 \\ Hongo, Bunkyo-Ku, Tokyo 113- \\ 8421, Japan. E-mail: y-uchi@ \\ juntendo.ac.jp.
}

\begin{abstract}
Toll-like receptors (TLRs) are one of the main contributors that induce inflammation under tissue injury and infection. Because excessive inflammation can aggravate disease states, it is important to control inflammation at a moderate level. Herein, we show that hyaluronan (HA) oligomer, HA tetrasaccharide (HA4), could suppress the expression of proinflammatory cytokine IL-1 $\beta$ when stimulated with both TLR2- and TLR4-specific agonists in primary hippocampal neurons. To understand the effect of HA4 against ischemic insult, we performed hypoxic-ischemic (H/I) brain injury against neonatal mice. HA4 treatment significantly prevented hippocampal pyramidal cell death even 7 days after H/I injury, compared with the control mice. Although TLR2 and TLR4 are known as receptors for HA and also act as a receptor for inducing inflammation, only TLR2-deficient mice showed tolerance against $\mathrm{H} / \mathrm{I}$ injury. Moreover, HA4 administration suppressed gliosis by inhibiting the activation of NF- $\kappa \mathrm{B}$, the downstream target of TLR2, which led to the suppression of IL-1 $1 \beta$ expression. Taken together, our data suggest that the neuroprotective effect of HA4 relies on antagonizing the TLR2/NF- $\kappa B$ pathway to reduce inflammation through suppressing the expression of proinflammatory cytokines after neonatal $\mathrm{H} / \mathrm{I}$ brain injury. (Am J Pathol 2016, 186: 2143-2151; http://dx.doi.org/10.1016/j.ajpath.2016.03.016)
\end{abstract}

Hypoxic-ischemic (H/I) brain injury in the perinatal period is known to link to subsequent neurological complications, such as seizure, cerebral palsy, mental retardation, and epilepsy. ${ }^{1}$ As recognized in neonates, symptomatic perinatal stroke occurs in one in 4000 live births. ${ }^{2}$ Morphological, biochemical, and genetic approaches have revealed that both apoptotic and nonapoptotic neuronal death takes place in the pyramidal layer of the hippocampus after $\mathrm{H} / \mathrm{I}$ injury. ${ }^{3-5}$ Oxidative stress and glutamate excitotoxicity are suspected to be major executors that lead to cell death in the initial stage. ${ }^{1}$ However, reducing inflammation could prevent the progression of damage in the following step. ${ }^{6}$ An understanding of the molecular pathways underlying neuronal cell death induced by neonatal $\mathrm{H} / \mathrm{I}$ brain injury could pave the way for new therapeutic approaches.
Toll-like receptors (TLRs) are the key receptors that initiate innate immune responses by recognizing specific molecular structures of pathogens known as pathogenassociated molecular patterns. ${ }^{7}$ However, TLRs are also stimulated by endogenous intracellular molecules released by activated or necrotic cells and extracellular matrix molecules that are up-regulated on injury or degraded after tissue damage, namely damage-associated molecular

Supported by Japan Society for the Promotion of Science Kakenhi grants 23390041 and 23659102 (Y.U.) and grants 23790239 and 26860139 (T.S.); the Scientific Research on Innovative Areas from the Ministry of Education, Science, Sports and Culture grants 23111004 and 23110517 (Y.U.); the MEXT-supported Program for the Strategic Research Foundation at Private Universities grant S1101009 (Y.U. and T.S.); and the Juntendo University Project Research grant-in-aid 2317 (T.S.).

Disclosures: Y.O. is employed by Glycoscience Laboratories Inc. 
patterns (DAMPs), even in the absence of infection. ${ }^{8,9}$ Accumulation of DAMPs initiates positive feedback loops of inflammation that increases tissue damage after impairment. Therefore, regulating the TLR signaling pathway is important for managing pathological conditions.

Although hyaluronan (HA) is known as one of the DAMPs that stimulates TLR2 and TLR4, ${ }^{10,11}$ interestingly, the activity of HA depends on size. In general, high-molecularweight-HA (HMW-HA; $6 \times 10^{5}$ to $1 \times 10^{6} \mathrm{Da}$ ) is antiangiogenic, anti-inflammatory, and immunosuppressive, whereas low-molecular-weight-HA (LMW-HA; $<5 \times 10^{4}$ Da) is inflammatory, immunostimulatory, and angiogenic. ${ }^{12}$ However, the definition of LMW-HA is ambiguous and little is known about small HA oligomers.

Herein, we revealed the effect of a small HA oligomer, HA tetrasaccharide (HA4; $820.24 \mathrm{Da}$ ) against neonatal H/I brain injury. Several recent articles reported that HA4 treatment has improvement in a symptom after spinal cord injury $^{13}$ or experimental autoimmune encephalomyelitis. ${ }^{14}$ However, little is known about the underlying molecular mechanisms. To further elucidate the effects of HA4 after neonatal $\mathrm{H} / \mathrm{I}$ brain injury, we focused on the relationship between TLRs and the induction of inflammation.

\section{Materials and Methods}

\section{Animals}

The procedures involving animal care, surgery, and sample preparation were approved by the Animal Experimental Committee of Juntendo University (Tokyo, Japan) and performed in accordance with the Guide for the Care and Use of Laboratory Animals. ${ }^{15}$ Neonatal C57BL/6J mice were obtained from Japan SLC (Shizuoka, Japan), and the C57BL/6 background TLR2 and TLR4 knockout (KO) mice were purchased from Oriental BioService (Kyoto, Japan). Mice were subsequently housed under specific pathogen-free conditions with a 12-hour light-dark cycle at Juntendo University.

\section{Hypoxic-Ischemic Injury}

Neonatal $\mathrm{H} / \mathrm{I}$ brain injury was induced in mice on post-natal day 7 (P7), essentially according to the Rice-Vannucci model $^{16}$ with minor modifications. ${ }^{17}$ After the mice were deeply anesthetized with isoflurane (2\%), the left common carotid artery was dissected and ligated with silk sutures $(6 / 0)$, followed by a recovery for 1 hour at $37^{\circ} \mathrm{C}$. They were then placed in chambers maintained at $37^{\circ} \mathrm{C}$ with a flow purged with nitrogen gas to control oxygen concentration to $8 \%$ for 25 minutes. After hypoxic exposure, the pups were returned to their dams and were allowed to recover for 1, 3, 6, 12, and 24 hours, and 7 days. At each stage, the brains were processed for molecular, biochemical, and morphological analyses. This procedure resulted in brain injury in the ipsilateral hemisphere, consisting of cerebral infarction mainly in the hippocampus. ${ }^{17-19}$ Pups with severe damage in the cortex were eliminated from the experiment. Control littermates were neither operated on nor subjected to hypoxia.

\section{Preparation of Hyaluronan Tetrasaccharide}

Preparation of HA4 was as follows. Hyaluronan (Shiseido, Tokyo, Japan) was digested in $0.3 \mathrm{~mol} / \mathrm{L}$ methanesulfonic acid at $80^{\circ} \mathrm{C}$ for several hours (18 to 20 hours). The digest was diluted in water and then adjusted to a $\mathrm{pH}$ between 5 and 7 with sodium hydroxide before being loaded onto a column containing Dowex $1 \times 4$ anion exchange resin (Wako Chemical, Osaka, Japan; 200 to 400 mesh). The $1 \times 4$ column was washed with an aqueous $40 \mathrm{mmol} / \mathrm{L}$ sodium chloride solution, and HA4 was eluted with a linear gradient consisting of 40 to $100 \mathrm{mmol} / \mathrm{L}$ sodium chloride. The Dowex $1 \times 4$ purified HA4 was adjusted to between $\mathrm{pH}$ 4 and $\mathrm{pH} 5$ with $\mathrm{HCl}$, diluted with water, and loaded onto a column containing BioRad High Q (anion exchange) resin (Bio-Rad Laboratories, Hercules, CA). The High Q column was washed with water, and HA4 was eluted with $0.5 \mathrm{~mol} / \mathrm{L}$ sodium chloride. The $\mathrm{pH}$ of the eluted HA4 solution was adjusted to $\mathrm{pH}$ approximately 5 with $\mathrm{HCl}$, concentrated by rotary evaporation, and then desalted by passing it through a BioGel P2 size exclusion column (Bio-Rad Laboratories) using water as the eluant. The desalted HA4 was purified with a Dowex 50Wx4 column (Wako Chemical) in the acid form. This purified HA4 was passed through ultrafilter membranes with a molecular weight cutoff of $10,000 \mathrm{Da}$ (Merck Millipore, Darmstadt, Germany), concentrated, and then dried to a powder in a rotary evaporator. The purity of HA4 was determined by high-performance liquid chromatography.

\section{Hyaluronan Administration}

HA4 was administered i.p. to P6 mouse pups 12 hours before, and just before, $\mathrm{H} / \mathrm{I}$ injury at $\mathrm{P} 7$ with a concentration of $10 \mathrm{mg} / \mathrm{kg}$ body weight. Saline administration was performed against littermates or age-matched mice as a control.

\section{Sample Preparation for Histochemical and Morphological Analyses}

After $\mathrm{H} / \mathrm{I}$ injury, mice were deeply anesthetized and fixed by cardiac perfusion with $4 \%$ paraformaldehyde buffered with $0.1 \mathrm{~mol} / \mathrm{L}$ phosphate buffer $(\mathrm{pH} 7.2)$ containing $4 \%$ sucrose. Brain tissues were quickly removed from the mice and further immersed in the same fixative overnight at $4^{\circ} \mathrm{C}$. Samples processed for paraffin embedding were cut into sections $(4-\mu \mathrm{m}$ thick) with a semimotorized rotary microtome (RM2245; Leica, Wetzlar, Germany) and placed on silane-coated glass slides. For routine histological studies, paraffin sections were stained with hematoxylin and eosin.

\section{Immunoblot Analysis}

Each tissue was independently homogenized in RIPA buffer $[50 \mathrm{mmol} / \mathrm{L}$ Tris- $\mathrm{HCl}(\mathrm{pH} 7.6)$ with $150 \mathrm{mmol} / \mathrm{L}$ 
$\mathrm{NaCl}, 1 \% \mathrm{NP}-40,0.5 \%$ sodium deoxycholate, and $0.1 \%$ SDS] containing a protease inhibitor-cocktail (Nacalai, Kyoto, Japan) and a phosphatase inhibitor-cocktail (Nacalai). After centrifugation at $20,000 \times g$ for 10 minutes at $4^{\circ} \mathrm{C}$, the protein concentrations in the supernatants were determined using the BCA protein assay system (Pierce, Rockford, IL), and were used for immunoblotting. The lysates were eluted by boiling in loading buffer, separated by SDS-PAGE, and transferred to an Immobilon P-membrane (Millipore). The blots were probed with anti-NF- $\kappa \mathrm{B}$ p65 antibodies (1:1000 dilution; Cell Signaling Technology, Danvers, MA) and anti-phospho-NF- $\kappa$ B p65 (Ser536) antibodies (1:500; Cell Signaling Technology).

\section{Immunohistochemical Analysis}

Deparaffinized sections were incubated overnight at $4^{\circ} \mathrm{C}$ with anti-NeuN antibodies (1:200; Merck Millipore), anti-Iba1 antibodies (1:200; Wako), anti-Mac2 antibodies (1:200; Cedarlane, Burlington, NC), and anti-glial fibrillary acidic protein antibodies (1:200; Sigma, St. Louis, MO). Sections were further reacted with peroxidase-conjugated secondary antibodies at room temperature for 2 hours and washed three more times. To visualize the sections, they were reacted with peroxidase, using $0.0125 \% 3,3^{\prime}$-diaminobenzidine tetrahydrochloride and $0.002 \% \mathrm{H}_{2} \mathrm{O}_{2}$ in $0.05 \mathrm{~mol} / \mathrm{L}$ Tris- $\mathrm{HCl}$ buffer (pH 7.6) for 5 minutes. For immunofluorescence staining antimouse, anti-rabbit IgG Alexa Fluor 488 antibodies (Thermo Fisher Scientific, Rockford, IL), and anti-rat $\mathrm{Cy} 3$ antibodies (Jackson ImmunoResearch Laboratories, West Grove, PA) were used as secondary antibodies. Immunostained sections were observed using a BX50 (Olympus, Tokyo, Japan) and confocal laser scanning microscope FV1000 (Olympus).

\section{qPCR and RT-PCR}

For real-time quantitative PCR (qPCR) and RT-PCR analyses, total RNA was prepared using RNeasy (Qiagen, Hilden, Germany) and cDNA was synthesized using a PrimeScript master mix (Takara, Shiga, Japan), according to the manufacturer's recommendations. The expression level of IL- $1 \beta$ for qPCR was analyzed using a Thunderbird SYBR qPCR mix (Toyobo, Osaka, Japan) on thermal cycler dice (Takara). The primer pairs were as follows: IL-1 $\beta, 5^{\prime}$-ACCTGTGGCCTTGGGCCTCAAA- $3^{\prime}$ (forward) and 5'-TTGGGATCCACACTCTCCAGCTGC-3' (reverse); $\beta$-actin, 5'-GGTGGGCCGCCCTAGGCACCA-3' (forward) and $5^{\prime}$-TTGGCCTTAGGGTTCAGGGGG- $3^{\prime}$ (reverse). The expressions of TLR2, TLR4, and $\beta$-actin were analyzed using KOD-plus (Toyobo). The primer pairs were as follows: TLR2, $5^{\prime}$-TGGTGTCTGGAGTCTGCTGTG-3' (forward) and 5'-CGCTCCGTACGAAGTTCTCAG-3' (reverse); TLR4, $5^{\prime}$-CAGTGGTCAGTGTGATTGTGG-3' (forward) and $5^{\prime}$-TTCCTGGATGATGTTGGCAGC-3' (reverse); and $\beta$-actin, 5'-GGTGGGCCGCCCTAGGCACCA- $3^{\prime}$ (forward) and $5^{\prime}$-TTGGCCTTAGGGTTCAGGGGG-3' (reverse).

\section{Primary Cultures of Hippocampal Neurons}

Primary hippocampal neurons were prepared for culturing, as previously described. ${ }^{20}$ Briefly, the hippocampi were dissected and dissociated from 18-day-old fetal mice. Dissociated hippocampal neurons were plated on poly-Lornithine (Sigma) coated 6-well plates with 2\% B27 (Invitrogen, San Diego, CA) and $2 \mathrm{mmol} / \mathrm{L}$ L-glutamine (Nacalai) in Neurobasal medium (Invitrogen). Two days later, 10 $\mathrm{mmol} / \mathrm{L}$ cytosine arabinoside (Nacalai) was added to inhibit nonneuronal growth. After 14 days, hippocampal neurons were stimulated with $1 \mu \mathrm{g} / \mathrm{mL}$ Pam3CSK4 (Invivogen) and $1 \mu \mathrm{g} / \mathrm{mL}$ LPS-SM Ultrapure (Invivogen) in the presence ( $50 \mathrm{ng} / \mathrm{mL}, 5 \mu \mathrm{g} / \mathrm{mL}$ ) or absence of HA4 for 6 hours on day 14 .

\section{TUNEL Staining}

Terminal deoxynucleotidyl transferase (TdT)-mediated dUTP nick end labeling (TUNEL) staining was applied to deparaffinized sections as previously described. ${ }^{21}$ Briefly, sections were incubated with $100 \mathrm{U} / \mathrm{mL}$ TdT (Promega, Madison, WI) and $10 \mathrm{nmol} / \mathrm{L}$ biotinylated 16-2'-dUTP (Roche Diagnostics, Basel, Switzerland) in TdT buffer (100 $\mathrm{mmol} / \mathrm{L}$ sodium cacodylate, $\mathrm{pH} 7.0,1 \mathrm{mmol} / \mathrm{L}$ cobalt chloride, $50 \mathrm{mg} / \mathrm{mL}$ gelatin) in a humid atmosphere at $37^{\circ} \mathrm{C}$ for 1 hour, followed by further incubation with peroxidaseconjugated streptavidin (Vector Laboratories, Burlingame, CA) for 1 hour at room temperature. Staining for peroxidase was performed as stated above.

\section{Cell Counting}

The number of TUNEL-positive cells was counted in the whole pyramidal regions of the hippocampus, located in the same portion (bregma: $-1.82 \mathrm{~mm}$ of adult $\mathrm{C} 57 \mathrm{BL} / \mathrm{J}$ mice), according to the previous report. ${ }^{17}$

\section{Neuropathological Evaluation}

H/I injury and neuroprotection were evaluated by the number of TUNEL-positive (dead) cells in the pyramidal layers of the hippocampus 24 hours after H/I injury using brain sections corresponding to the same portion of the hippocampus as mentioned above. The damaged area in the hippocampal pyramidal layers was also examined 7 days after $\mathrm{H} / \mathrm{I}$ injury, in which time clear-cut shrinkage occurred in the ipsilateral hippocampus of the control mice, resulting in a loss of ipsilateral hippocampal neurons. To quantitate these damaged areas after neonatal H/I, the areas of lesioned and unlesioned hippocampi were measured and compared within the same section corresponding to the same portions mentioned above, using ImageJ software version 1.38 (NIH, Bethesda, MD). The area loss of the lesioned ipsilateral hippocampus was calculated as a percentage of the intact contralateral hippocampus, and was determined for each animal. 


\section{Statistical Analysis}

All quantified data were expressed as the means \pm SEM. Differences between two mean values were evaluated using an unpaired Student's $t$-test and two-way analysis of variance post-hoc tests that were considered statistically significant when the $P$ value was $<0.05$.

\section{Results}

\section{HA4 Antagonizes Both TLR2 and TLR4 Signaling Pathway in Primary Hippocampal Neurons}

The hippocampus is the brain region that is most vulnerable to cerebral ischemic insult, ${ }^{18,19}$ and Toll-like receptors (TLRs) are known to be involved in neuronal cell death after ischemia. $^{22-25}$ To determine the effect of a small HA oligomer, HA tetrasaccharide (HA4; 820.24 Da), we focused on the TLR signaling pathway for three reasons: i) TLR2 and TLR4 are known as the receptors for $\mathrm{HA}^{10,11}$; ii) stimulation of TLRs would activate NF- $\kappa \mathrm{B}$ and induce proinflammatory cytokines ${ }^{7}$; and iii) primary cortical neurons generated from TLR2-KO and TLR4-KO mice are resistant to glucose deprivation. ${ }^{22}$ Therefore, we first explored whether HA4 could participate in the TLR/NF- $\kappa \mathrm{B}$ signaling pathway in primary hippocampal neurons. To confirm the expressions of TLR2 and TLR4 in primary hippocampal neurons, we performed reverse transcriptasePCR. Primary hippocampal neurons cultured for 14 days in vitro expressed both TLR2 and TLR4 as well as the microglial cell line BV2 (Figure 1A). Thus, we stimulated TLR2 and TLR4 with specific agonists, Pam3CSK4 and LPS, respectively, and observed the expression of the proinflammatory cytokine IL- $1 \beta$ by qPCR in the presence and absence of HA4. The increased expression of IL- $1 \beta$ by Pam3CSK4 stimulation (2.7-fold) and LPS stimulation (2.8fold) was suppressed in a dose-dependent manner by HA4 administration ( $50 \mathrm{ng} / \mathrm{mL}, 5 \mu \mathrm{g} / \mathrm{mL}$ of HA4, respectively), whereas no change was observed when HA4 was administered alone (Figure 1B). These data suggest that HA4 can play an antagonistic role against TLR2 and TLR4 in the hippocampal neuron in vitro.

\section{HA4 Administration Protects Hippocampal Pyramidal Neurons from Neonatal H/I Injury}

To elucidate the effect that HA4 administration exerts against cerebral ischemic insult in vivo, we next examined the damage in the pyramidal regions of the hippocampus in neonatal mouse brains after H/I injury. HA4 $(10 \mathrm{mg} / \mathrm{kg}$ body weight) was administered i.p., 12 hours, and immediately before $\mathrm{H} / \mathrm{I}$ injury, and physiological saline was injected as a control. The degree of damage in the hippocampal pyramidal cells, quantified as the number of TUNEL-positive nuclei, was extremely reduced in mice treated with HA4, compared with those treated with saline

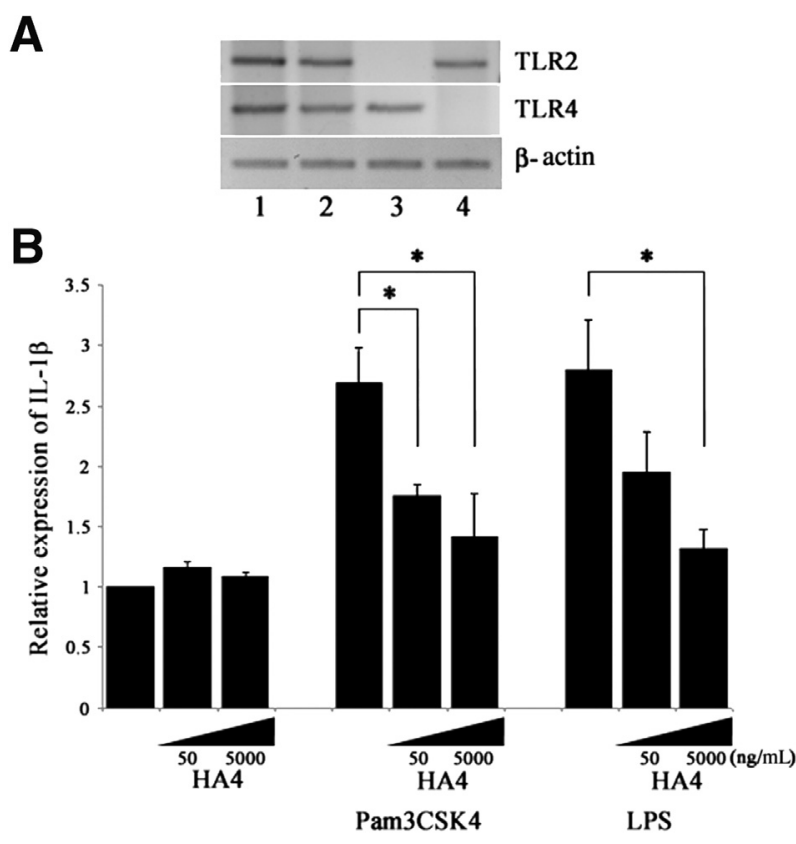

Figure 1 HA4 antagonizes both TLR2 and TLR4 signaling pathway in primary hippocampal neurons. A: Expression of TLR2 and TLR4 mRNA was confirmed by reverse transcriptase-PCR in lysates from BV2 cells (lane 1; a microglial cell line), primary hippocampal neurons (lane 2), TLR2-K0 mouse brain (lane 3), and TLR4-KO mouse brain (lane 4). B: Quantification of expression levels of IL-1 $\beta$ mRNA in primary hippocampal neurons cultured in the presence of Pam3CSK4 and lipopolysaccharide (LPS) with $50 \mathrm{ng} / \mathrm{mL}$ and $5 \mu \mathrm{g} / \mathrm{mL}$ of HA4 or without HA4, as measured by real-time quantitative PCR. Data are expressed as means \pm SEM (B). $n=4$ independent experiments (B). ${ }^{*} P<0.05$.

24 hours after H/I injury (Figure 2, A-D). The number of TUNEL-positive nuclei within the pyramidal layer of mouse brains (Figure 2, B and D) that were administered with HA4 was significantly decreased $(179 \pm 19)$ to fewer than half the number of those treated with saline $(377 \pm 51)$ (Figure 2E). We farther evaluated the number of TUNELpositive nuclei, in the CA1 and CA3 region of the hippocampal pyramidal cell layer. The number of TUNELpositive nuclei within CA1 region (Figure $2 \mathrm{~F}$ ) significantly decreased by HA 4 administration $(44 \pm 10)$ compared with saline $(214 \pm 30)$. However, although slight reduction of the number of TUNEL-positive nuclei was also observed, no significance was detected between HA4 $(17 \pm 6)$ and saline $(61 \pm 24)$ administration in the CA3 region (Figure $2 \mathrm{~F})$. These data suggest that HA4 administration can protect hippocampal pyramidal cells after neonatal $\mathrm{H} / \mathrm{I}$ brain injury in a region-specific manner.

Pathological Alterations Are Not Detected in the Hippocampal Pyramidal Neurons of a HA4-Administered Neonatal Hippocampus 7 Days after H/I Injury

Delayed neuronal death reportedly occurs in the pyramidal layers of the hippocampus within 7 days after transient brain ischemia, and damaged areas are replaced by glial cells. ${ }^{19}$ 


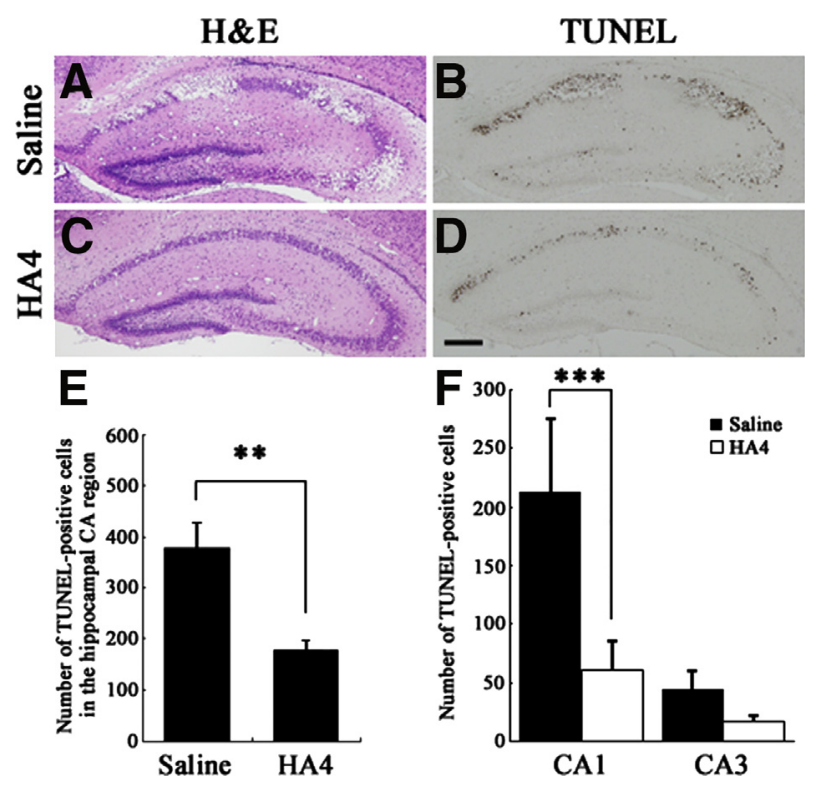

Figure 2 HA4 administration protects hippocampal pyramidal cells from neonatal $\mathrm{H} / \mathrm{I}$ injury. Representative demonstration of the ipsilateral hippocampi of mice administered saline ( $\mathbf{A}$ and $\mathbf{B})$ and HA4 (C and $\mathbf{D})$, respectively, stained with hematoxylin and eosin (H\&E; $\mathbf{A}$ and $\mathbf{C}$ ) and for terminal deoxynucleotidyl transferase-mediated dUTP nick end labeling (TUNEL; B and D) 24 hours after H/I injury. E: Quantitative analysis of the number of TUNELpositive cells in the pyramidal layer of an identical portion (bregma: -1.82 $\mathrm{mm}$ of adult $\mathrm{C} 57 \mathrm{BL} / \mathrm{J}$ mice) of the hippocampus, administered saline and HA4, 24 hours after H/I injury. F: Quantitative analysis of the number of TUNEL-positive cells in the CA1 and CA3 region of an identical portion (bregma: $-1.82 \mathrm{~mm}$ of adult $\mathrm{C} 57 \mathrm{BL} / \mathrm{J}$ mice) of the hippocampus, administered saline and HA4, 24 hours after $\mathrm{H} / \mathrm{I}$ injury. Data are expressed as means \pm SEM (E and $\mathbf{F}) . n=13$ and 14 mice per group $(\mathbf{E}) ; n=8$ and 6 mice per group $(\mathbf{F}) .{ }^{* *} P<0.01,{ }^{* *} P<0.001$. Scale bar $=200 \mu \mathrm{m}(\mathbf{A}-\mathbf{D})$.

To exclude the possibility that the administration of HA4 simply delayed the onset of pyramidal cell death, we further analyzed the degree of damage in the neurons of the hippocampal pyramidal layers in HA4-administered and control littermate mice 7 days after H/I injury. Loss of NeuN-positive neurons with their neurites that was eliminated by microglial cells contributed to the areal reduction of pyramidal layers of the control mouse hippocampus (Figure 3, C and D). The total area of the ipsilateral hippocampus was significantly diminished, compared with the contralateral hippocampus (Figure 3, A and B). Meanwhile, in the HA4-administered mice, the remaining pyramidal neurons were largely TUNEL-negative (data not shown), and staining for hematoxylin and eosin, and NeuN showed those neurons to be intact (Figure 3, A-F). The ipsilateral hippocampal area in the HA4-administered mice was only slightly diminished to $96 \% \pm 9 \%$ compared with the contralateral hippocampi (Figure 3, B and G). However, in the physiological saline-injected littermate controls, the ipsilateral hippocampal area was reduced to nearly half the contralateral hippocampal area 7 days after $\mathrm{H} / \mathrm{I}$ injury $(53 \% \pm 7 \%)$ (Figure 3, A and G). These data indicate that the prevention of hippocampal pyramidal cell death by HA4 administration is sustained at least until 7 days after $\mathrm{H} / \mathrm{I}$ injury.

The Neuroprotective Effect of HA4 Largely Relies on the Inhibition of TLR2, but Not TLR4 in Neonatal H/I Injury

HA4 antagonized both TLR2 and TLR4 in primary hippocampal neurons in vitro (Figure 1) and protected hippocampal pyramidal cells after $\mathrm{H} / \mathrm{I}$ injury in vivo (Figures 2 and 3). However, the major target of the neuroprotective role of HA4 after neonatal H/I injury remained unclear. To confirm the relationship between TLR2, TLR4, and HA4 in vivo, we used TLR2-KO and TLR4-KO mice and applied them to $\mathrm{H} / \mathrm{I}$ injury. When saline was administered, the degree of damage in the hippocampal pyramidal cells was extremely reduced in TLR2-KO mice (Figure 4C), compared
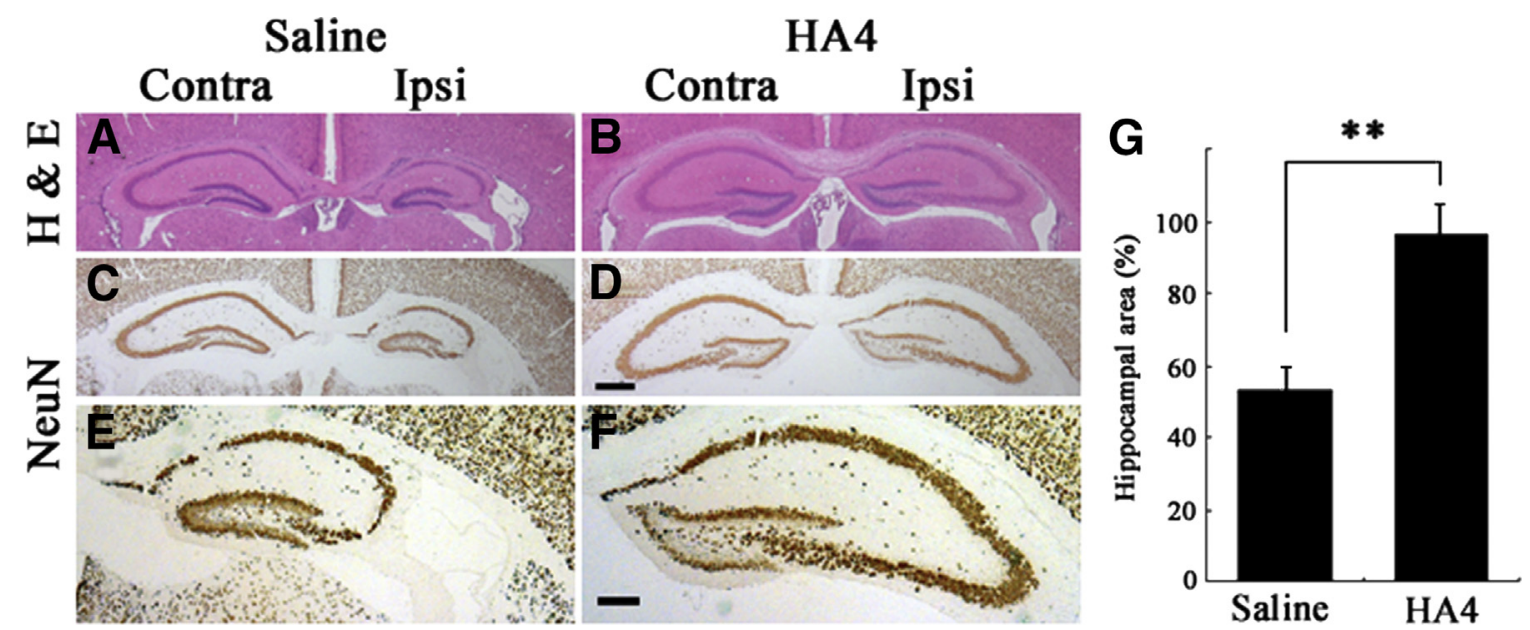

Figure 3 Prevention of neuronal loss in the hippocampus by HA4 administration is further sustained up to 7 days after $\mathrm{H} / \mathrm{I}$ injury. Representative demonstration of the hippocampi of mice administered saline (A, C, and $\mathbf{E})$ or HA4 (B, D, and $\mathbf{F})$ stained with hematoxylin and eosin (H\&E; A and $\mathbf{B})$ and antiNeuN antibodies (C-F) 7 days after H/I injury. G: Evaluation of the area of ipsilateral (Ipsi) against contralateral (Contra) hippocampi of HA4-treated and littermate control mouse brains of an identical portion (bregma: $-1.82 \mathrm{~mm}$ of adult $\mathrm{C} 57 \mathrm{BL} / \mathrm{J}$ mice) of the hippocampus 7 days after $\mathrm{H} / \mathrm{I}$ injury. Data are expressed as means \pm SEM (G). $n=9$ and 7 mice per group (G). ${ }^{* *} P<0.01$. Scale bars: $400 \mu \mathrm{m}(\mathbf{A}-\mathbf{D}) ; 200 \mu \mathrm{m}(\mathbf{E}$ and $\mathbf{F})$. 


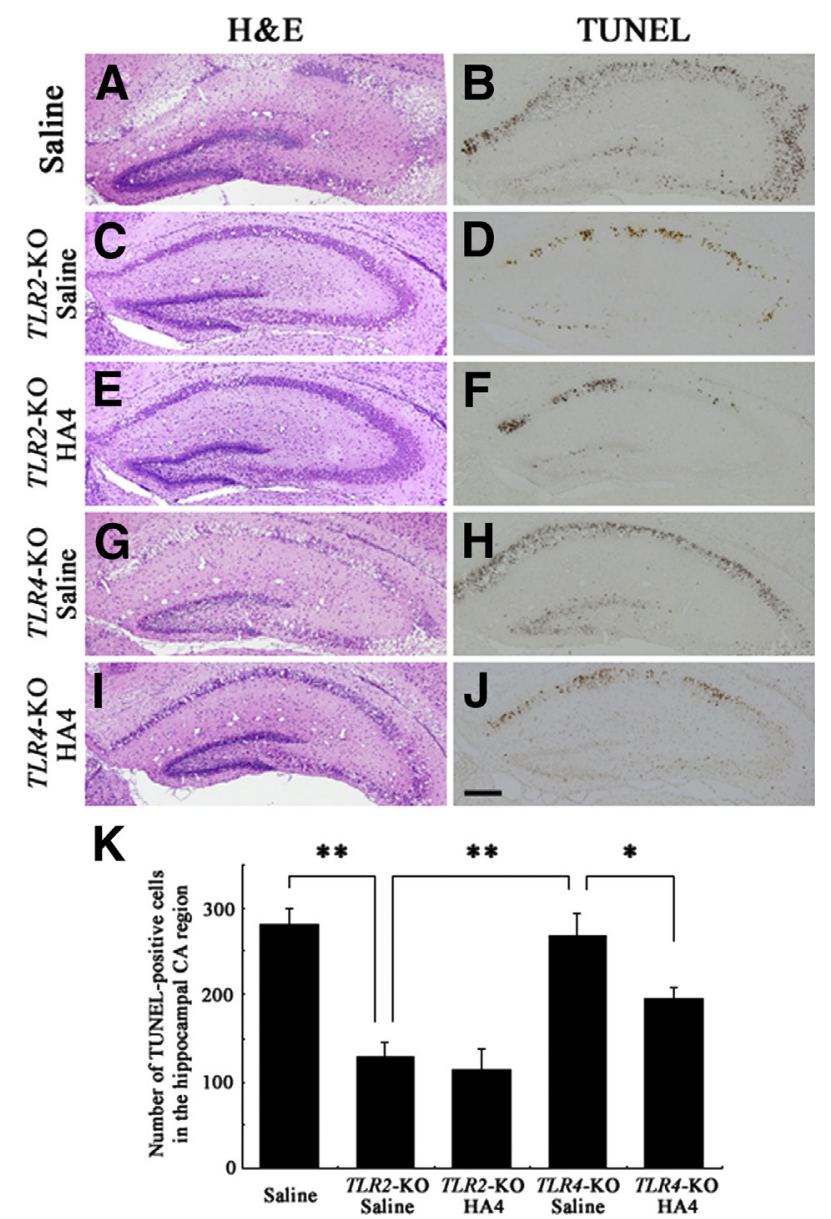

Figure 4 TLR2 is the major target for the neuroprotective effect of HA4 after $\mathrm{H} / \mathrm{I}$ injury in vivo. Representative demonstration of the ipsilateral hippocampus of wild-type (A and $\mathbf{B}), T L R 2-K O(\mathbf{C}-\mathbf{F})$, and TLR4-KO (G-J) mice administered saline (A-D, G, and $\mathbf{H})$ or $\mathrm{HA} 4(\mathbf{E}, \mathbf{F}, \mathbf{I}$, and $\mathbf{J})$, and obtained 24 hours after $\mathrm{H} / \mathrm{I}$ injury. A, C, E, G, and I: Hematoyxlin and eosin (H\&E) staining. B, D, F, H, and J: Terminal deoxynucleotidyl transferase-mediated dUTP nick end labeling (TUNEL) staining. K: Quantification of the number of TUNELpositive cells in the pyramidal layer of an identical portion (bregma: -1.82 $\mathrm{mm}$ of adult $\mathrm{C} 57 \mathrm{BL} / \mathrm{J}$ mice) of the hippocampus of wild-type, TLR2-KO, and TLR4-KO mice administered saline or HA4 and obtained 24 hours after $\mathrm{H} / \mathrm{I}$ injury. Data are expressed as means $\pm \operatorname{SEM}(\mathrm{K}) . n=6$ or 7 mice per group (K). ${ }^{*} P<0.05,{ }^{*} P<0.01$. Scale bar $=200 \mu \mathrm{m}(\mathbf{A}-\mathbf{J})$.

with both TLR4-KO (Figure 4G) and wild-type mice (Figure 4A) 24 hours after H/I injury, which was consistent with previous reports. ${ }^{23,24}$ Moreover, the improvement after administration of HA4 was observed only in TLR4-KO mice, but not in TLR2-KO mice (Figure 4, E and I). The TUNEL-positive cells within the ipsilateral hippocampal pyramidal layer (Figure 4, B, D, F, H, and J) were significantly decreased in TLR2-KO mice (130 \pm 16$)$, compared with wild-type mice $(280 \pm 19)$, and no additional improvement was observed after the administration of HA4 $(113 \pm 24)$ (Figure $4 \mathrm{~K})$. In contrast, the TUNEL-positive cells in TLR4-KO mice (268 \pm 26$)$ showed comparable level to the control mice, whereas the TUNEL positivity was significantly suppressed and the insult was improved by HA4 administration (194 \pm 15 ) (Figure $4 \mathrm{~K}$ ). Taken together, these data indicate that the major target of HA4 after neonatal H/I brain injury was TLR2 but not TLR4.

HA4 Administration Inhibits the Expression of Proinflammatory Cytokine IL-1 $\beta$ through Inactivating $\mathrm{NF}-\kappa \mathrm{B}$ after $\mathrm{H} / \mathrm{I}$ Injury

$\mathrm{H} / \mathrm{I}$ injury induces an inflammatory reaction in the neonatal brain, and the early proinflammatory phase is thought to deteriorate pathological states. ${ }^{6}$ Inhibition of proinflammatory cytokines, such as IL- $1 \beta^{26}$ and IL- $18,{ }^{27}$ and the activator of IL-1 and IL-18, caspase- $1,{ }^{28}$ reduces H/I brain injury to a moderate degree. Therefore, we evaluated the expression level of IL-1 $\beta$ in the ipsilateral hippocampus with or without HA4 administration and compared with TLR2-KO mice by qPCR. The expression level of IL- $1 \beta$ increased within an hour and peaked 6 hours after $\mathrm{H} / \mathrm{I}$ injury in the control mice (Figure 5A). However, HA4 administration, as well as TLR2-KO mice, significantly inhibited IL-1 $\beta$ expression 3 and 6 hours after $\mathrm{H} / \mathrm{I}$ injury (Figure $5 \mathrm{~A}$ ). To further confirm the molecular mechanism regulating the expression of IL-1 $\beta$, we next verified the activity of the NF- $\kappa B / R e l$ family. The Ser536 residue of p65/RelA, a member of the NF- $\kappa \mathrm{B} / \mathrm{Rel}$ family, is phosphorylated when $\mathrm{NF}-\kappa \mathrm{B}$ is activated and translocates to the nucleus to regulate gene expression including IL- $1 \beta .^{29}$ Western blot analyses revealed that the activation of NF- $\kappa \mathrm{B}$ in the ipsilateral hippocampi, represented as an expression ratio of phospho-p65/RelA (Ser536) per pan-p65/RelA, increased 5.2-fold within an hour in control mice when compared with sham-operated mice, whereas the activation was significantly suppressed to 1.4-fold by HA4 administration and 0.98-fold in TLR2-KO mice (Figure 5, B and C). These data indicate that HA4 administration can inhibit the expression of proinflammatory cytokine IL-1 $\beta$ in vivo, in a closely related manner to TLR2-KO mice, by inactivating NF- $\kappa \mathrm{B}$ in the early stages after H/I Injury.

\section{HA4 Administration Inhibits Gliosis after H/I Injury}

Although the expression of IL-1 $\beta$ mRNA was suppressed by HA4 administration (Figure 5), the activation (cleavage for the secretion form) of IL-1 $\beta$, which causes inflammation, was undetectable by both Western blot analyses and enzyme-linked immunosorbent assay analyses after H/I brain injury. Therefore, we focused on gliosis as the hallmark of inflammation. Gliosis consists of the activation of astrocytes and microglial cells, stimulated by proinflammatory cytokines, including IL-1 $\beta$. The activation of microglial cells and astrocytes was investigated by evaluating the expression of Mac2 (for reactive microglial cells), Iba1 (for pan-microglial cells), and glial fibrillary acidic protein (for reactive astrocytes) 24 hours after H/I injury (Figure 6). Interestingly, the Iba1-positive microglial cells slightly increased the number and many coexpressed Mac2 in the control hippocampus (Figure 6A), whereas HA4 


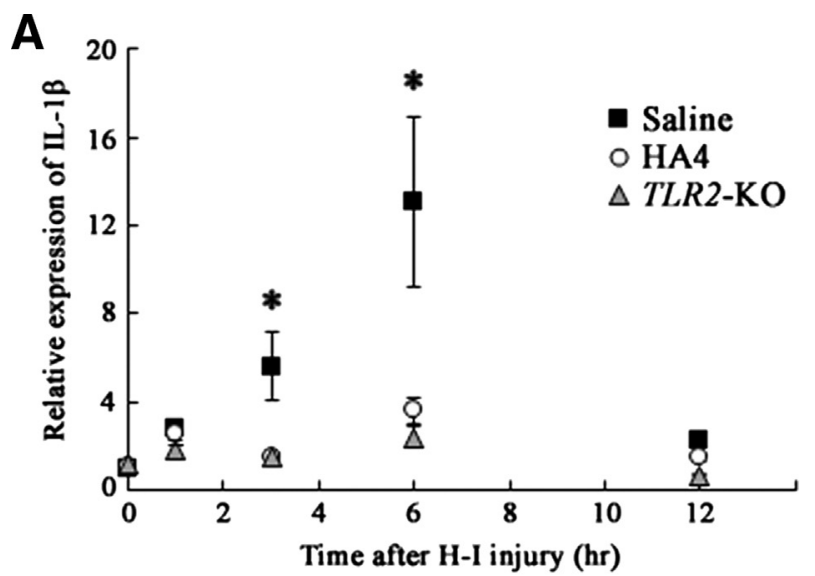

B
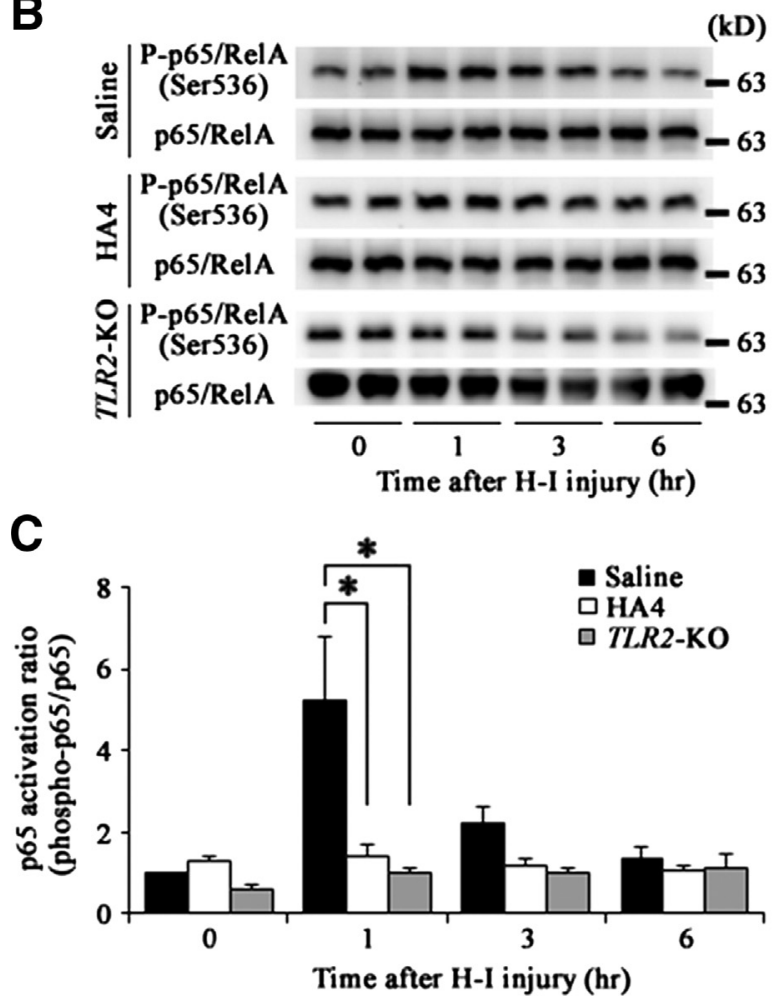

Figure 5 Inhibitory effect of HA4 on the expression of a proinflammatory cytokine, IL-1 $\beta$, through inactivation of NF- $\mathrm{KB}$ after $\mathrm{H} / \mathrm{I}$ injury. A: Quantification of IL-1 $\beta$ mRNA levels in ipsilateral hippocampi obtained from HA4- or saline-treated and TLR2-KO mice at indicated time periods after $\mathrm{H} / \mathrm{I}$ injury. mRNA levels were measured by real-time quantitative PCR. B: Western blot analyses of phospho-p65/RelA (Ser536) and pan-p65/RelA in the ipsilateral hippocampi from HA4- or saline-administered and TLR2-KO mouse brains. Duplicated data are presented in protein extracts from the brains of mice injected with HA4 or saline and TLR2-KO. C: Ratios of protein amounts of phospho-p65/RelA (Ser536)/pan-p65/RelA in the ipsilateral hippocampi administered HA4 or saline and TLR2-KO. Data are expressed as means \pm SEM (A and C). $n=3$ to 7 mice per group (A); $n=4$ mice per group $(\mathbf{C}) .{ }^{*} P<0.05$.

administration diminished the expression of Iba1 to the steady state and Mac2-expressing cells were not observed (Figure 6B). Moreover, the expression of glial fibrillary acidic protein was strongly elevated only in the control hippocampus, suggesting that HA4 administration suppressed the emergence of reactive astrocytes (Figure 6, C and D). Taken together, these data indicate that HA4 administration protects neuronal cell death through suppressing inflammation via inhibiting the expression of proinflammatory cytokine IL-1 $\beta$.

\section{Discussion}

Inflammation is induced by both tissue injury and infection for facilitating wound healing and stimulating innate immunity. However, excessive inflammation can also aggravate symptoms. Although lines of evidence from KO animals have revealed that TLRs are one of the main contributors not only to pathogen-induced inflammation, but also to injury-induced inflammation in various organs,${ }^{30}$ the strategies to attenuate inflammation after tissue damage have been limited. Herein, we demonstrated the following five points: i) HA4 exhibited antagonistic action against both TLR2 and TLR4 in vitro; ii) HA4 administration prevented hippocampal pyramidal neuron death for at least 7 days after neonatal $\mathrm{H} / \mathrm{I}$ brain injury; iii) the main target of this inhibition was TLR2 but not TLR4 in vivo; iv) HA4 administration decreased the expression of proinflammatory cytokine IL- $1 \beta$ by inactivating the NF- $\kappa \mathrm{B} / \mathrm{Rel}$ family; and v) HA4 administration prevented inflammation.

HA has a high molecular mass $\left(>10^{6} \mathrm{Da}\right)$ under physiological conditions. However, in inflammatory tissues, the activation of hyaluronidase degrades HMW-HA into small

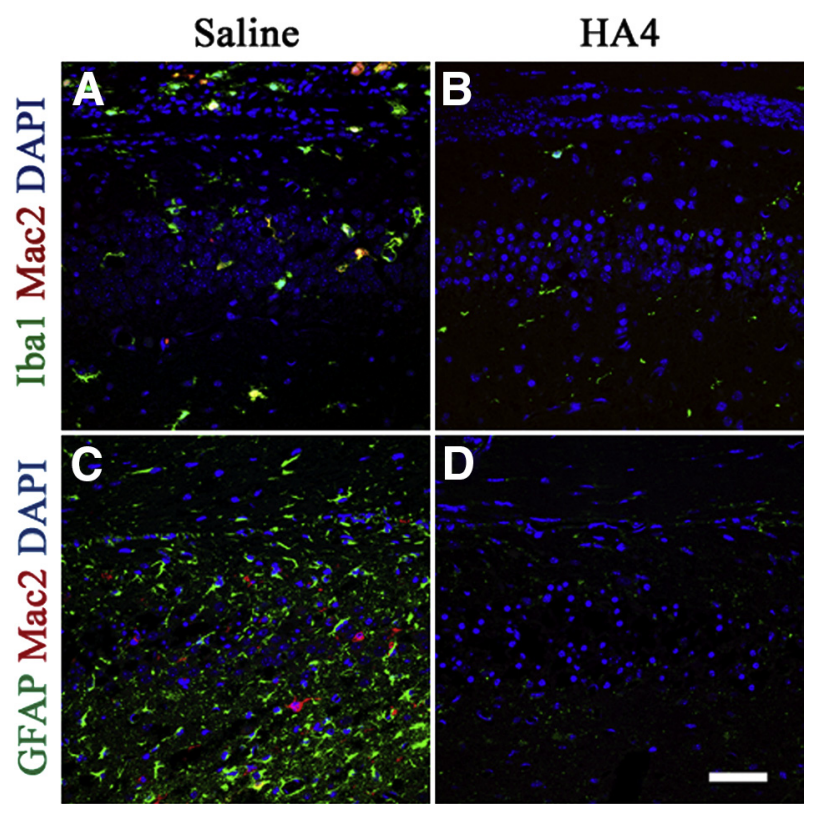

Figure 6 The emergence of Mac2-expressing microglial cells and reactive astrocytes is suppressed by HA4 administration 24 hours after $\mathrm{H} / \mathrm{I}$ injury. Representative demonstration of the ipsilateral hippocampi administered saline (A and $\mathbf{C}$ ) and HA4 (B and $\mathbf{D})$ stained with anti-Iba1 antibodies (green; $\mathbf{A}$ and $\mathbf{B}$ ), anti-glial fibrillary acidic protein (GFAP) antibodies (green; $\mathbf{C}$ and $\mathbf{D}$ ), anti-Mac2 antibodies (red; $\mathbf{A}-\mathbf{D}$ ), and DAPI (blue; A-D). Scale bar $=50 \mu \mathrm{m}(\mathbf{A}-\mathbf{D})$. 
fragments, whereas increased free radicals also depolymerize HMW-HA. ${ }^{31,32}$ Such fragmented or depolymerized LMW-HA molecules ( 1 to $2.5 \times 10^{4} \mathrm{Da}$ ) are known to be potent stimulators of inflammatory cytokines through TLR2 and TLR4. During acute lung injury, depolymerized LMWHA ( 1.35 to $\left.2 \times 10^{4} \mathrm{Da}\right)$ fragments will cause inflammation through both TLR2 and TLR4 in vivo. ${ }^{33}$ Conversely, forced expression of HMW-HA $\left(>10^{6} \mathrm{Da}\right)$ exerts protective effects on lung injury and epithelial cell apoptosis. ${ }^{33}$ Our finding that the small HA oligomer (HA4) administration protected neurons from $\mathrm{H} / \mathrm{I}$ injury (Figures 2 and 3) may explain the diverse biological effects of HA and its fragments.

Our present data suggested that the neuroprotective effect of HA4 results from the antagonistic action against TLR to prevent the expression of proinflammatory cytokine IL- $1 \beta$ that leads to inflammation (Figures 1, 5, and 6). Our hypothesis concerning how HA4 can antagonize the TLR/ NF- $\kappa \mathrm{B}$ pathway is as follows. Because HA is a straight chain of nonsulfated glycosaminoglycan that is composed of repeating polymeric disaccharides, D-glucuronic acid and $\mathrm{N}$-acetyl glucosamine, it is plausible that HA4 can be accessible to TLR2 and TLR4 as well as LMW-HA. Ligandinduced receptor dimerization of TLR is a crucial step to launch the cascade that finally results in the activation of several transcription factors, including NF- $\kappa \mathrm{B}{ }^{7}$ We speculate that HA4 binds to TLR2 and TLR4 and fills the pocket to block the binding of stimulants, but that it is too small to couple the receptors to activate the cascade. Therefore, HA4 can suppress the expression of proinflammatory cytokines by inactivating the TLR/NF- $\kappa B$ pathway. Consistent with our hypothesis, several groups have suggested that HA oligomers could attenuate the hyaluronan signaling by displacing multivalent HA polymers and inhibiting the malignant progression in various kinds of cancer. ${ }^{34}$

We have previously reported that HA4 up-regulates the expression of Hsp72 (an inducible form of Hsp70) and heat shock factor-1, and that it can prevent cell death under serum deprivation of neuronally differentiated PC12 cells. ${ }^{35}$ In the current study, we did not observe the expression of Hsp72. However, it is known that overexpression of Hsp70 attenuates brain damage after permanent and transient middle cerebral artery occlusion by reducing inflammation in adult mice. ${ }^{36,37}$ In addition, overexpression of Hsp72 decreases the release of IL-1 $\beta$ when macrophages were stimulated with high mobility group box $1 .^{38}$ Although the regulatory mechanism is yet unclear, we will not exclude the possibility that the recovery observed by HA4 administration is because of an increase in Hsp70 expression.

The number of TUNEL-positive nuclei dramatically decreased by HA4 administration throughout the entire hippocampal pyramidal layer (Figure 2E), and the CA1 region showed comparable results (Figure 2F). However, despite the slight reduction ( $61 \pm 24$ to $17 \pm 6$ by HA4 administration) (Figure 2F), no significant differences were observed in the $\mathrm{CA} 3$ region. Because the number of TUNEL-positive nuclei were limited and distinction between samples was extensive, one interpretation could be the insufficiency of samples; however, this could be also rationalized by the difference of the cell death mode between CA1 and CA3 pyramidal cells. ${ }^{5}$

TLR2 and TLR4 are both known to be involved in neuronal cell death after cerebral ischemia-reperfusion in adults. ${ }^{25}$ In addition, HA4 administration antagonized specific agonists against both TLR2 and TLR4 in primary hippocampal neurons (Figure 1). However, the neonatal H/I injury model revealed that the major target of the neuroprotective role of HA4 was TLR2 but not TLR4 (Figure 4). This discrepancy could be because of the expression patterns of TLR2 and TLR4 in vitro and in vivo. Although we confirmed that TLR2 and TLR4 were both expressed in primary hippocampal neurons and the neonatal hippocampus by reverse transcriptase-PCR (Figure 1), we could not quantify the precise expression amounts of either TLR2 or TLR4, nor could we determine which cells were responsible for the promotion of inflammation. However, because TLR4-KO mice showed no significant recovery against the control mice after H/I injury, HA4 administration and TLR2KO could protect hippocampal pyramidal cell death to the equivalent level (Figure 4), it is possible to predict that the expression of TLR4 has little effect on hippocampal neuronal cell death after neonatal $\mathrm{H} / \mathrm{I}$ injury.

A recent report has shown that the peroxiredoxin family proteins released extracellularly after brain ischemiareperfusion injury in adult mice stimulate TLR2 and TLR4 as a DAMP. ${ }^{25}$ Interestingly, the blocking antibodies attenuated ischemic brain damage and suppressed inflammatory cytokine expression when systemically administered even 12 hours after ischemia. ${ }^{25}$ These data suggest that targeting TLRs could expand the therapeutic window after stroke. A large body of evidence suggests that TLR2 and/or TLR4 play a pivotal role in ischemia-reperfusion injuries in various organs (ie, hepatic, renal, cerebral, intestinal, and myocardial). ${ }^{30}$ Thus, HA4 could be an attractive candidate for clinical applications in ischemic insult. Moreover, although the recurrence rate after perinatal stroke is lower than in older children and adults, ${ }^{2}$ prophylactic efficacy of HA4 could be expected against stroke in newborn infants.

\section{References}

1. Ferriero DM: Neonatal brain injury. N Engl J Med 2004, 351: 1985-1995

2. Nelson KB, Lynch JK: Stroke in newborn infants. Lancet Neurol 2004 , 3:150-158

3. Sheldon RA, Hall JJ, Noble LJ, Ferriero DM: Delayed cell death in neonatal mouse hippocampus from hypoxia-ischemia is neither apoptotic nor necrotic. Neurosci Lett 2001, 304:165-168

4. Uchiyama Y, Koike M, Shibata M: Autophagic neuron death in neonatal brain ischemia/hypoxia. Autophagy 2008, 4:404-408

5. Ginet V, Puyal J, Clarke PG, Truttmann AC: Enhancement of autophagic flux after neonatal cerebral hypoxia-ischemia and its regionspecific relationship to apoptotic mechanisms. Am J Pathol 2009, 175:1962-1974 
6. Hagberg H, Gressens P, Mallard C: Inflammation during fetal and neonatal life: implications for neurologic and neuropsychiatric disease in children and adults. Ann Neurol 2012, 71:444-457

7. Kumar H, Kawai T, Akira S: Pathogen recognition in the innate immune response. Biochem J 2009, 420:1-16

8. Bianchi ME: DAMPs, PAMPs and alarmins: all we need to know about danger. J Leukoc Biol 2007, 81:1-5

9. Piccinini AM, Midwood KS: DAMPening inflammation by modulating TLR signalling. Mediators Inflamm 2010, 2010. pii:672395

10. Scheibner KA, Lutz MA, Boodoo S, Fenton MJ, Powell JD, Horton MR: Hyaluronan fragments act as an endogenous danger signal by engaging TLR2. J Immunol 2006, 177:1272-1281

11. Termeer C, Benedix F, Sleeman J, Fieber C, Voith U, Ahrens T, Miyake K, Freudenberg M, Galanos C, Simon JC: Oligosaccharides of Hyaluronan activate dendritic cells via toll-like receptor 4. J Exp Med 2002, 195:99-111

12. Day AJ, de la Motte CA: Hyaluronan cross-linking: a protective mechanism in inflammation? Trends Immunol 2005, 26:637-643

13. Wakao N, Imagama S, Zhang H, Tauchi R, Muramoto A, Natori T, Takeshita S, Ishiguro N, Matsuyama Y, Kadomatsu K: Hyaluronan oligosaccharides promote functional recovery after spinal cord injury in rats. Neurosci Lett 2011, 488:299-304

14. Winkler CW, Foster SC, Itakura A, Matsumoto SG, Asari A, McCarty OJ, Sherman LS: Hyaluronan oligosaccharides perturb lymphocyte slow rolling on brain vascular endothelial cells: implications for inflammatory demyelinating disease. Matrix Biol 2013, 32: $160-168$

15. Committee for the Update of the Guide for the Care and Use of Laboratory AnimalsNational Research Council: Guide for the Care and Use of Laboratory Animals. Eighth Edition. Washington, DC, National Academies Press, 2011

16. Rice JE, Vannucci RC, Brierley JB: The influence of immaturity on hypoxic-ischemic brain damage in the rat. Ann Neurol 1981, 9: 131-141

17. Koike M, Shibata M, Tadakoshi M, Gotoh K, Komatsu M, Waguri S, Kawahara N, Kuida K, Nagata S, Kominami E, Tanaka K, Uchiyama Y: Inhibition of autophagy prevents hippocampal pyramidal neuron death after hypoxic-ischemic injury. Am J Pathol 2008, 172: 454-469

18. Sheldon RA, Sedik C, Ferriero DM: Strain-related brain injury in neonatal mice subjected to hypoxia-ischemia. Brain Res 1998, 810: $114-122$

19. Ness JM, Harvey CA, Strasser A, Bouillet P, Klocke BJ, Roth KA: Selective involvement of BH3-only Bcl-2 family members Bim and Bad in neonatal hypoxia-ischemia. Brain Res 2006, 1099: $150-159$

20. Kaech S, Banker G: Culturing hippocampal neurons. Nat Protoc 2006, 1:2406-2415

21. Nitatori T, Sato N, Waguri S, Karasawa Y, Araki H, Shibanai K, Kominami E, Uchiyama Y: Delayed neuronal death in the CA1 pyramidal cell layer of the gerbil hippocampus following transient ischemia is apoptosis. J Neurosci 1995, 15:1001-1011

22. Tang SC, Arumugam TV, Xu X, Cheng A, Mughal MR, Jo DG, Lathia JD, Siler DA, Chigurupati S, Ouyang X, Magnus T, Camandola S, Mattson MP: Pivotal role for neuronal Toll-like receptors in ischemic brain injury and functional deficits. Proc Natl Acad Sci U S A 2007, 104:13798-13803

23. Stridh L, Smith PL, Naylor AS, Wang X, Mallard C: Regulation of toll-like receptor 1 and -2 in neonatal mice brains after hypoxiaischemia. J Neuroinflammation 2011, 8:45

24. Mallard C, Wang X, Hagberg H: The role of Toll-like receptors in perinatal brain injury. Clin Perinatol 2009, 36:763-772, v-vi

25. Shichita T, Hasegawa E, Kimura A, Morita R, Sakaguchi R, Takada I, Sekiya T, Ooboshi H, Kitazono T, Yanagawa T, Ishii T, Takahashi H, Mori S, Nishibori M, Kuroda K, Akira S, Miyake K, Yoshimura A: Peroxiredoxin family proteins are key initiators of post-ischemic inflammation in the brain. Nat Med 2012, 18:911-917

26. Hagberg H, Gilland E, Bona E, Hanson LA, Hahin-Zoric M, Blennow M, Holst M, McRae A, Söder O: Enhanced expression of interleukin (IL)-1 and IL-6 messenger RNA and bioactive protein after hypoxia-ischemia in neonatal rats. Pediatr Res 1996, 40:603-609

27. Hedtjärn $\mathrm{M}$, Leverin $\mathrm{AL}$, Eriksson $\mathrm{K}$, Blomgren $\mathrm{K}$, Mallard $\mathrm{C}$, Hagberg H: Interleukin-18 involvement in hypoxic-ischemic brain injury. J Neurosci 2002, 22:5910-5919

28. Liu XH, Kwon D, Schielke GP, Yang GY, Silverstein FS, Barks JD: Mice deficient in interleukin-1 converting enzyme are resistant to neonatal hypoxic-ischemic brain damage. J Cereb Blood Flow Metab 1999, 19:1099-1108

29. Baeuerle PA, Henkel T: Function and activation of NF-kappa B in the immune system. Annu Rev Immunol 1994, 12:141-179

30. Arumugam TV, Okun E, Tang SC, Thundyil J, Taylor SM, Woodruff TM: Toll-like receptors in ischemia-reperfusion injury. Shock 2009, 32:4-16

31. Fraser JR, Laurent TC, Laurent UB: Hyaluronan: its nature, distribution, functions and turnover. J Intern Med 1997, 242:27-33

32. Deguine V, Menasche M, Ferrari P, Fraisse L, Pouliquen Y, Robert L: Free radical depolymerization of hyaluronan by Maillard reaction products: role in liquefaction of aging vitreous. Int J Biol Macromol 1998, 22:17-22

33. Jiang D, Liang J, Fan J, Yu S, Chen S, Luo Y, Prestwich GD, Mascarenhas MM, Garg HG, Quinn DA, Homer RJ, Goldstein DR, Bucala R, Lee PJ, Medzhitov R, Noble PW: Regulation of lung injury and repair by Toll-like receptors and hyaluronan. Nat Med 2005, 11: 1173-1179

34. Toole BP: Hyaluronan: from extracellular glue to pericellular cue. Nat Rev Cancer 2004, 4:528-539

35. $\mathrm{Xu} \mathrm{H}$, Ito $\mathrm{T}$, Tawada $\mathrm{A}$, Maeda $\mathrm{H}$, Yamanokuchi $\mathrm{H}$, Isahara $\mathrm{K}$, Yoshida K, Uchiyama Y, Asari A: Effect of hyaluronan oligosaccharides on the expression of heat shock protein 72. J Biol Chem 2002, 277:17308-17314

36. Rajdev S, Hara K, Kokubo Y, Mestril R, Dillmann W, Weinstein PR, Sharp FR: Mice overexpressing rat heat shock protein 70 are protected against cerebral infarction. Ann Neurol 2000, 47:782-791

37. Zheng Z, Kim JY, Ma H, Lee JE, Yenari MA: Anti-inflammatory effects of the $70 \mathrm{kDa}$ heat shock protein in experimental stroke. J Cereb Blood Flow Metab 2008, 28:53-63

38. Tang D, Kang R, Xiao W, Wang H, Calderwood SK, Xiao X: The antiinflammatory effects of heat shock protein 72 involve inhibition of high-mobility-group box 1 release and proinflammatory function in macrophages. J Immunol 2007, 179:1236-1244 